\title{
Mathematical modelling of electrical potential difference in a non-uniform electric field
}

\author{
Mustafa Erol ${ }^{a}$, ildahan Özdeyiş Çolak ${ }^{b}$ \\ Dokuz Eylül University. Cumhuriyet Blv. No:144, 35220 Alsancak, Konak,İzmir,Turkey \\ a mustafa.erol@deu.edu.tr, bozdeyiscolak@gmail.com \\ * Corresponding Author.
}

\begin{abstract}
This work offers an unproblematic teaching tool for the instruction of challenging concept of electric potential difference in a non-uniform electric field. Specifically, mathematical modelling process is employed and managed to comprehend and teach exceedingly difficult concepts of uniform and non-uniform electric fields, electrical potential difference, scalar products of vectors and also concept of path integral. In order to accomplish those tasks, initially a basic conducting panel/sheet, that is simply a wet cardboard, is designed as a part of the apparatus, together with a dc power supply, a multi meter and connecting cables. The established method is interesting in the sense that the 3D wet cardboard is novel, very practical and minimal costing, hence the approach offers physics educators fresh teaching routes and opportunities to clarify the puzzling concept of electrical potential difference and further.
\end{abstract}

Keywords: electrical potential difference; mathematical modelling; non-uniform electric field; path integral; physics education.

How to Cite: Erol, M., \& Çolak, Illdahan Özdeyiş. (2020). Mathematical modelling of electrical potential difference in a non-uniform electric field. Momentum: Physics Education Journal, 4(2), 64-72. https://doi.org/10.21067/mpej.v4i2.4440

\section{Introduction}

Physics resolves the nature and related phenomena on the base of decidedly complicated concepts and mathematical equations between those concepts. Teaching those concepts, phenomena and mathematical equations is the fundamental issue of Physics Education Research (PER). Physics educators, around the globe, spend much effort to ease teaching complicated and so believed abstract concepts and equations such that every single student manages conceptual understanding and internalises the actual scientific knowledge (Retnawati et al., 2018; Zhang et al., 2018).

In contrast to classical mechanics, electricity and also magnetism are, for vast majority of students, found to be abstract and confusing subjects of physics needing more consideration and effort for an enhanced understanding (Afra et al., 2009; Hand et al., 2009; Moodley \& Gaigher, 2019; Taber et al., 2006). Therefore, teaching electricity and magnetism faces more problematic circumstances and ought to be handled more wisely (Gunstone et al., 2009; Kelly et al., 1998; Martinez et al., 2011; Tarciso Borges \& Gilbert, 1999). Teaching electrical potential and potential difference, in a non-uniform electric field, is a challenging task due to be containing an integral equation. The actual mathematical equation includes the fundamental concepts of uniform or non-uniform electric field, electrical potential difference together with the distance within the electrical field and also a path integral therefore, instructing the general form of potential difference have been a puzzling task for physics educators and teachers (Carlton, 1999; Cohen et al., 1983).

An analysis of the conceptual structure of physics, on the other hand, identifies necessary factual and procedural knowledge which is not plainly formulated and taught in courses. It leads to the conclusion that mathematical modelling of the natural phenomena should be the central theme 
of physics education (Hestenes, 1987). Scientific equations are, in general, coherent units of structured knowledge. They are used to establish factual information into coherent wholes, often by the harmonised use of general laws or principles (Hestenes, 1997). The physics instruction is centred on the idea that physicists reason from mental constructs known as models. Scientists resolve the natural phenomena by using tools such as graphs, charts, diagrams and eventually reach to mathematical equations to represent definite physical laws or principles (Brewe, 2008). A model, in general, is a substitute object or mental constructs, hence a conceptual representation of a real entity. The models, in physics education, are mostly mathematical equations, that means physical properties are characterised by quantifiable variables in the models (Hestenes, 1987). Students, on the other hand, learn exchangeable mathematical modelling skills by applying given mathematical equations to a range of situations to describe or predict physical events or to design experiments. Nevertheless, in an old-style physics class students do not have a clear understanding of what the word 'model' means and thus do not appreciate the role of this notion in physics instruction (Grosslight et al., 1991). The significances of a 'modelling view of physics for physics teaching, would be that physics education should give students a view of the nature of physics as a mathematical modelling creativity. Modelling accordingly receive increasing consideration from the physics educators as important components of a trendy physics education (Gilbert, 2004; Gilbert et al., 2000; Greca \& Moreira, 2002).

The students permanently find it difficult to understand and internalise the uniform and nonuniform electric fields, the potential differences within those electric fields and surely the path independence of the potential differences together with the actual path integrals (Liégeois et al., 2003; Maloney et al., 2001; Prosser, 1994; Rosenthal \& Henderson, 2006). Therefore, the present work focuses on resolving apparent teaching difficulties concerning the electrical potential difference and consequently the path integrals, simply based on three fundamental problem cases which are expressed next; (1) How can one explore and teach, on a simple 3D material, the mathematical relation between the electrical potential change and the actual electric field on a straight line within a uniform electric field?; (2) How can one explore and teach, on a simple 3D material, whether the sum of the potential differences is path dependent or not, within a non-uniform electric field?; and (3) How can one explore and teach, on a simple 3D material, the mathematical relation between the electrical potential difference and the actual electric field on a randomly curved path, within a nonuniform electric field?

The present work specifically focuses on resolving apparent complications on instructing the intricate concept of electric potential difference. Therefore, the effort aims to develop a priceless and basic 3D material to demonstrate and teach the confusing concept of electrical potential difference. Following the search of the 3D teaching material, mathematical modelling method is employed to drive the fundamental equation of potential difference. The material is very advantageous in the sense that it costs nothing and works exceptionally well.

\section{Method}

\section{Teaching Material}

One of the fundamental aims of this work, was to develop a low-cost 3D material, that could be easily found in any school around the globe and easy to use. To manage this task, following a number of attempts and failures, a simple smooth cardboard is finally developed and employed throughout the modelling. The cardboard has a thickness of about $1 \mathrm{~mm}$ and dimensions of $17 \mathrm{~cm} \times 11.5 \mathrm{~cm}$. The cardboard, when dry, is a good insulator due to on its cellulosic nanostructure and could not be employed for the modelling since no potential differences could be measured across. Knowing the fact that the water is actually a good electrical conductor, made us to combine the cardboard with water. Therefore, dry smooth cardboard is simply immersed in an appropriate tap water tank for only 1 second and obtained homogeneously distributed water molecules and in fact a wet cardboard. The electrical conductance of evenly wet cardboard is used as an opportunity 
to teach. The chemical properties of the water, such as distillation or ionization energy are not within the scope of the work. The photography of the scaled wet cardboard is given in the Figure 1.

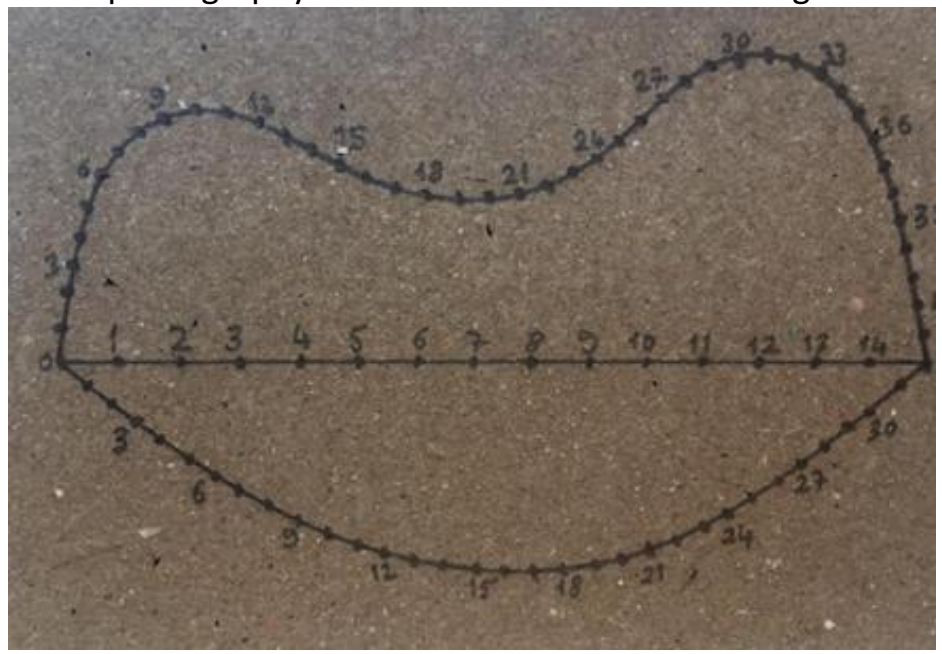

Figure 1. The photography of the wet scaled cardboard used for the experiment

In the Figure 1, we also show the 3 different paths, namely 1st path that is the straight one, 2nd path that is almost circular one and 3rd path that is the randomly chosen one. The paths and the basic scaling on the cardboard are managed before the immersing process on the dry cardboard in order not to damage the actual surface.

\section{Experimental Details}

The apparatus employed for the work and actual mathematical modelling of electrical potential difference is shown in the Figure 2 . The experimental setup is made up of a homogeneously wet and scaled cardboard, a DC power supply (max.18 V), a number of connection cables and a basic multimeter. During the actual experiment, a DC voltage of $15 \mathrm{~V}$ is applied to the two ends of the wet cardboard, however due to the contact problems, a DC voltage of $10.18 \mathrm{~V}$ is measured between the actual measurement points that are the points $A$ and $B$. The apparent difference is attributed to the well-known and unavoidable contact problem and does not affect the proposed teaching activities by any means.

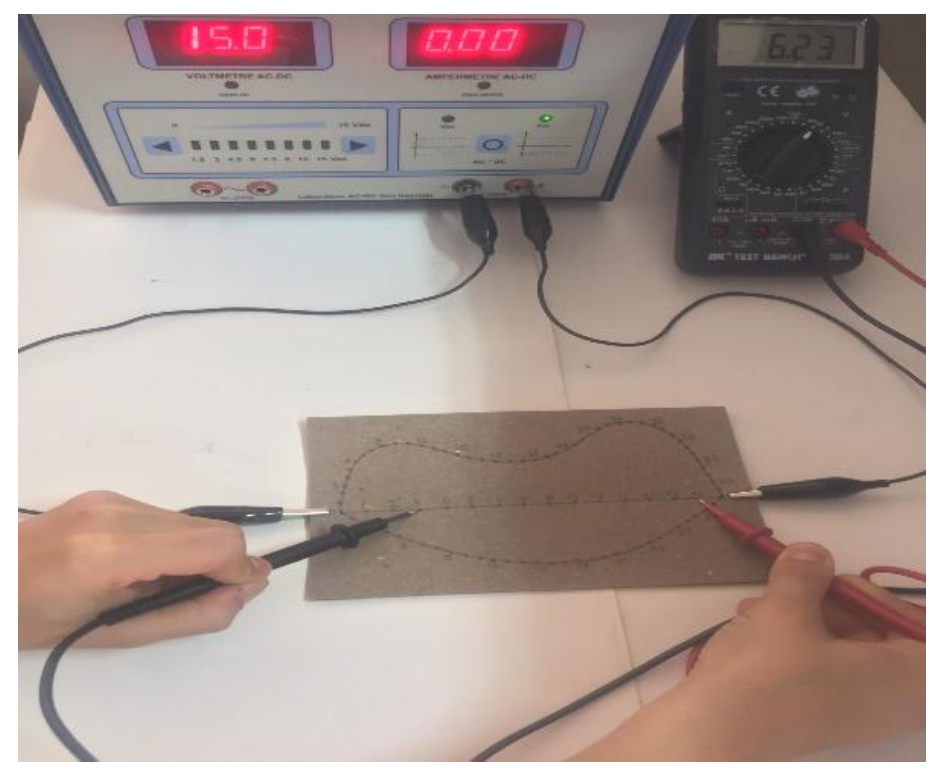

Figure 2. The photography of the experimental setup, also showing an instantaneous measurement

In order to answer the research problem statements, the following procedure is accomplished. For the first problem statement, basically the potential difference is measured, with respect to the 
point of $A$, as a function of the distance on the 1st path that is the straight path. For the second problem statement, the potential differences, this time, are sequentially measured as a function of the $0.5 \mathrm{~cm}$ long path intervals for the three paths, namely straight path, almost circular path and randomly chosen path. To manage the 3rd problem statement, the experimental data obtained for the 2 nd problem case is simply engaged.

\section{Results and Discussion}

\section{The potential difference and distance relation in a uniform electric field}

In order to tackle the first problem statement, we searched the mathematical relation between the electrical potential change and the actual electric field within a uniform electric field created on a straight line. The electric field is assumed to be uniform due to the fact that the external voltage of $15 \mathrm{~V}$ is directly applied across the straight path, in other words between the points of $A$ and $B$, due also the fact that the electric field vectors and displacement vectors are parallel and in addition to the charge density along the path ought to be uniform. To do so, we set the distance to the starting point, $A$, as the free variable and the voltage, $V$, as the dependant variable and simply measured the potential difference as a function of the distance. The actual measurements are given in the Table 1. The actual results, used to obtain the mathematical modelling between the uniform electric field and the potential difference, is presented in the Table 1 . The direct measurement of the potential difference between the two points, $A$ and $B$, is found to be, $V_{B}-V_{A}=10,18 \mathrm{~V}$.

Table 1. The measured potential differences as a function of the distance to the starting point, that is $A$, for the straight path

\begin{tabular}{|c|c|c|c|c|c|}
\hline$x(\mathrm{~cm})$ & V (volt) & $x(\mathrm{~cm})$ & V (volt) & $x(\mathrm{~cm})$ & V (volt) \\
\hline 1 & 1.15 & 7 & 4.87 & 13 & 8.22 \\
\hline 2 & 1.98 & 8 & 5.44 & 14 & 8.96 \\
\hline 3 & 2.63 & 9 & 5.97 & 15 & 10.18 \\
\hline 4 & 3.31 & 10 & 6.5 & & \\
\hline 5 & 3.81 & 11 & 7.01 & & \\
\hline 6 & 4.4 & 12 & 7.6 & & \\
\hline
\end{tabular}

\section{Path dependence of the electric potential difference}

The second problem statement is about the relation between the sum of electrical potential differences and the actual shape of the path, within a uniform and also non-uniform electric fields. In order to search the path dependence of the potential difference, we simply measured the potential differences sequentially between every $1 \mathrm{~cm}$ long path intervals for the straight path (1st path) and between every $0.5 \mathrm{~cm}$ path intervals for the almost circular (2nd path) path and randomly chosen (3rd path) paths. The reason to measure the voltage differences across $0.5 \mathrm{~cm}$ for the curved paths (2nd path and 3rd path) is to reduce the nonlinearity effect, in order words reducing displacement intervals surely improves the accuracy of the measurements. The measurements are given in the tables 2, 3 and 4. Table 2 is for the first path, Table 2 is for the almost circular path and finally table 3 is for the randomly chosen path. The direct measurement of the potential difference between the points $\mathrm{A}$ and $\mathrm{B}$ is same again, $V_{B}-V_{A}=10.18 \mathrm{~V}$.

Table 2. The sequentially measured potential differences as a function of the $1 \mathrm{~cm}$ long path intervals for the straight path. The sum of the potential differences gives, $\Sigma \Delta V=9,76 \mathrm{~V}$

\begin{tabular}{cccccc}
\hline$\Delta \mathbf{x}(\mathbf{c m})$ & $\Delta \mathbf{V}(\mathbf{V})$ & $\Delta \mathbf{x}(\mathbf{c m})$ & $\Delta \mathbf{V}(\mathbf{V})$ & $\Delta \mathbf{x}(\mathbf{c m})$ & $\Delta \mathbf{V}(\mathrm{V})$ \\
\hline $\mathbf{0 - 1}$ & 1.22 & $\mathbf{6 - 7}$ & 0.44 & $\mathbf{1 2 - 1 3}$ & 0.56 \\
$\mathbf{1 - 2}$ & 0.81 & $\mathbf{7 - 8}$ & 0.49 & $\mathbf{1 3 - 1 4}$ & 0.71 \\
$\mathbf{2 - 3}$ & 0.64 & $\mathbf{8 - 9}$ & 0.51 & $\mathbf{1 4 - 1 5}$ & 1.28 \\
$\mathbf{3 - 4}$ & 0.59 & $\mathbf{9 - 1 0}$ & 0.45 & & \\
$\mathbf{4 - 5}$ & 0.52 & $\mathbf{1 0 - 1 1}$ & 0.50 & & \\
$\mathbf{5 - 6}$ & 0.53 & $\mathbf{1 1 - 1 2}$ & 0.51 & & \\
\hline
\end{tabular}


Momentum: Physics Education Journal, 4 (2), 2020, 68

Mustafa Erol, i̇ldahan Özdeyiş Çolak

Table 3. The sequentially measured potential differences as a function of the $0.5 \mathrm{~cm}$ long path intervals for the almost circular path. The sum of the potential differences gives, $\sum \Delta V=9,55 \mathrm{~V}$.

\begin{tabular}{|c|c|c|c|c|c|c|c|}
\hline$\Delta x(\mathrm{~cm})$ & $\Delta V(V)$ & $\Delta x(\mathrm{~cm})$ & $\Delta V(V)$ & $\Delta x(\mathrm{~cm})$ & $\Delta V(V)$ & $\Delta x(\mathrm{~cm})$ & $\Delta V(V)$ \\
\hline $0-0.5$ & 0.69 & $2.5-3$ & 0.32 & $5-5.5$ & 0.22 & $7.5-8$ & 0.23 \\
\hline $0.5-1$ & 0.57 & 3-3.5 & 0.24 & $5.5-6$ & 0.21 & $8-8.5$ & 0.20 \\
\hline $1-1.5$ & 0.41 & $3.5-4$ & 0.30 & $6-6.5$ & 0.21 & $8.5-9$ & 0.23 \\
\hline $1.5-2$ & 0.31 & 4-4.5 & 0.28 & 6.5-7 & 0.21 & $9-9.5$ & 0.24 \\
\hline $2-2.5$ & 0.40 & 4.5-5 & 0.26 & $7-7.5$ & 0.19 & $9.5-10$ & 0.22 \\
\hline$\Delta x(\mathrm{~cm})$ & & & $\Delta x(\mathrm{~cm})$ & $\Delta V(V)$ & & & $\Delta V(V)$ \\
\hline $10-10.5$ & & & $12.5-13$ & 0.21 & & & 0.60 \\
\hline $10.5-11$ & & & $13-13.5$ & 0.22 & & & 0.78 \\
\hline $11-11.5$ & & & 13.5-14 & 0.27 & & & \\
\hline $11.5-12$ & & & $14-14.5$ & 0.30 & & & \\
\hline $12-12.5$ & & & 14.5-15 & 0.27 & & & \\
\hline
\end{tabular}

Table 4. The sequentially measured potential differences as a function of the $0.5 \mathrm{~cm}$ long path intervals for the randomly drawn path. The sum of the potential differences gives, $\Sigma \Delta V=10,40 \mathrm{~V}$.

\begin{tabular}{|c|c|c|c|c|c|c|c|c|c|}
\hline$\Delta x(\mathrm{~cm})$ & $\Delta V(V)$ & $\Delta x(\mathrm{~cm})$ & $\Delta V(V)$ & $\Delta x(\mathrm{~cm})$ & $\Delta V(V)$ & $\Delta x(\mathrm{~cm})$ & $\Delta V(V)$ & $\Delta x(\mathrm{~cm})$ & $\Delta V(V)$ \\
\hline $0-0.5$ & 0.55 & $2.5-3$ & 0.23 & $5-5.5$ & 0.18 & $7.5-8$ & 0.21 & $10-10.5$ & 0.21 \\
\hline $0.5-1$ & 0.60 & $3-3.5$ & 0.20 & $5.5-6$ & 0.17 & $8-8.5$ & 0.23 & $10.5-11$ & 0.22 \\
\hline $1-1.5$ & 0.21 & $3.5-4$ & 0.17 & $6-6.5$ & 0.15 & $8.5-9$ & 0.27 & $11-11.5$ & 0.23 \\
\hline $1.5-2$ & 0.36 & 4-4.5 & 0.16 & $6.5-7$ & 0.18 & $9-9.5$ & 0.29 & $11.5-12$ & 0.18 \\
\hline $2-2.5$ & 0.21 & 4.5-5 & 0.16 & 7-7.5 & 0.19 & $9.5-10$ & 0.27 & $12-12.5$ & 0.18 \\
\hline$\Delta x(\mathrm{~cm})$ & $\Delta V(V)$ & & $\Delta x(\mathrm{~cm})$ & $\Delta V(V)$ & $\Delta x(\mathrm{~cm})$ & $\Delta V(V)$ & & $\Delta x(\mathrm{~cm})$ & $\Delta V(V)$ \\
\hline $12.5-13$ & 0.17 & & $15-15.5$ & 0.21 & $17.5-18$ & 0.15 & & $20-20.5$ & 0.37 \\
\hline $13-13.5$ & 0.15 & & $15.5-16$ & 0.17 & $18-18.5$ & 0.17 & & $20.5-21$ & 0.42 \\
\hline $13.5-14$ & 0.16 & & $16-16.5$ & 0.16 & 18.5-19 & 0.18 & & $21-21.5$ & 0.65 \\
\hline $14-14.5$ & 0.18 & & $16.5-17$ & 0.14 & 19-19.5 & 0.22 & & $21.5-22$ & 0.30 \\
\hline $14.5-15$ & 0.17 & & $17-17.5$ & 0.16 & $19.5-20$ & 0.26 & & & \\
\hline
\end{tabular}

Relation between the electric field and potential difference in a non-uniform electric field

The third problem statement deals with the mathematical relation between the electrical potential difference and the non-uniform electric field strength, independent of the actual shape of the path. This part of the work simply uses the data in both Tables 3 and 4 . The actual mathematical modelling is about to be performed at this stage. The mathematical equation between the potential difference and the distance between those points, the electrical field and the actual path integral is scientifically and carefully derived at this stage, which is managed in the following section of the paper.

\section{Mathematical Modelling}

\section{Modelling the relation between the potential difference and uniform electric field}

In order to tackle with the first problem statement, we search for the mathematical relation between the electrical potential change and the actual electric field within a uniform electric field. For this case, the data in the Table 1 is to be used. The independent variable of the mathematical modelling is determined as the distance to the starting point, that is $A$, and the dependant variable is the actual voltage measured. In order to model the scientific law, the measured voltage is plotted as a function of the distance and the graph is given in the Figure 3.

It is clearly seen from the Figure 3 that the relation is linear and the relation is simply curve fitted by the equation of $V(x)=0.5896 x(\mathrm{~cm})+0.715$. This expression shows a linear relation between the voltage and the distance in accordance with the Equation 1. Since the relation between voltage, $V$, and distance, $x$, is linear than it is straightforward to write the actual slope as, 
$\tan \theta=\frac{\Delta V(V)}{\Delta x(\mathrm{~cm})}=0,5896 \frac{\mathrm{V}}{\mathrm{cm}}$, where $\theta$ denotes the angle of the fit with respect to the horizontal. It is now open to name the actual slope as the Electric Field; E, be simply looking at the unit and the dimension analysis of the equation. Finally, it can be stated that, the relation between the electric field and the potential difference when the displacement vector and electric vectors are parallel, can be given by,

$\Delta V=E \Delta x$

This is the result of the mathematical modelling for the 1st problem case and underlines one of the most fundamental laws of electricity (Beichner \& Serway, 2000; Sears et al., 2016).

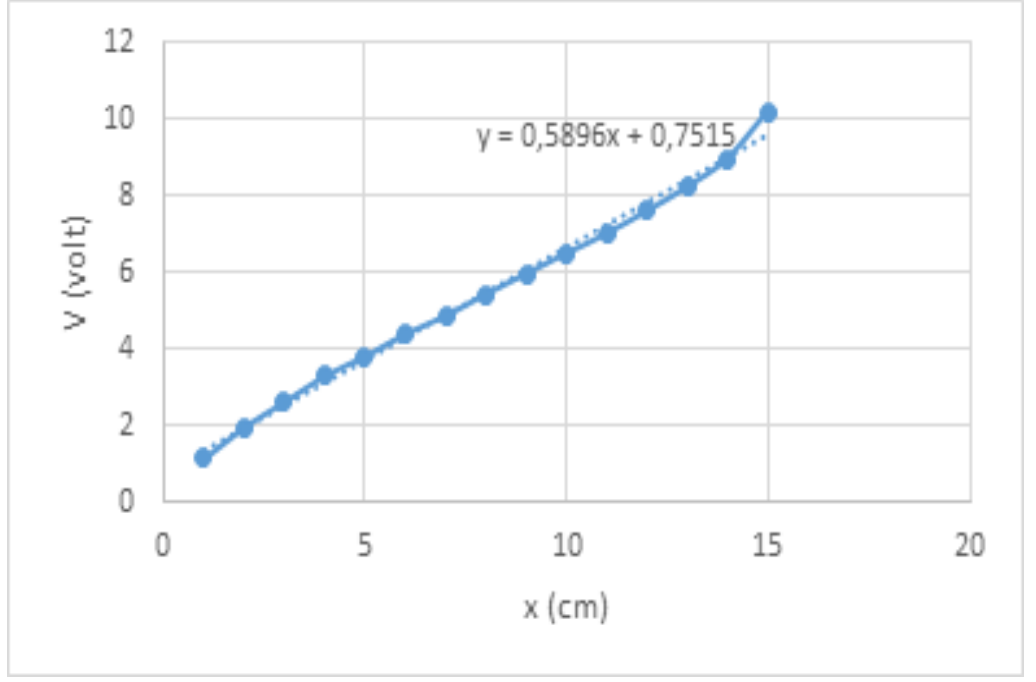

Figure 3. The measured voltage is plotted as a function of the distance for the linear path

\section{Modelling path dependence of the electric potential difference}

In order to focus on the second problem statement, three different paths connecting the points $A$ and $B$, that are the actual points external voltage is applied in between, are determined and sketched. The straight path is scaled by $1 \mathrm{~cm}$ path intervals, the almost circular path and randomly drawn paths are scaled by $0.5 \mathrm{~cm}$ path intervals. The actual measurements in between those points are performed sequentially by a multimeter and recorded in the tables 2,3 , and 4 . The electric field on the cardboard is obviously not uniform and changes point by point. Specifically, in this case the sums are given as $9.76 \mathrm{~V}$ for the straight path, $9.55 \mathrm{~V}$ for the second path and 10,40V for the third path. The discrepancies between the direct measurement and the overall sum voltages are about 4,1 $\%$ for the first path, $6,1 \%$ for the second path and finally $2.1 \%$ for the third path. These values are thought to be acceptable within the experimental error limits. The clear answer to the second problem statement is that the potential difference in between any two points in a non-uniform electric field is free of the actual path (Beichner \& Serway, 2000; Sears et al., 2016).

The sum of the measured voltages noticeably determines the answer of the second problem case, in the sense that if the sums for the three different paths are same then one can conclude that the electrical potential difference is independent of the path, which can mathematically be expressed by,

$V_{B}-V_{A}=\Sigma_{n} \Delta V_{n}$

Modelling relation between the electric potential difference and electric field in a non-uniform electric field

In order to resolve the third problem statement, the data given in the tables 2, 3, and 4 are used. The schematic drawing in the Figure 4 can be used for the actual modelling. The figure 4 demonstrates the 1st linear path, the 2nd almost circular path and 3rd randomly drawn path in addition to the electric field and displacement vectors. The angle between the actual electric field vectors and the displacement vectors is denoted by $\theta$. 


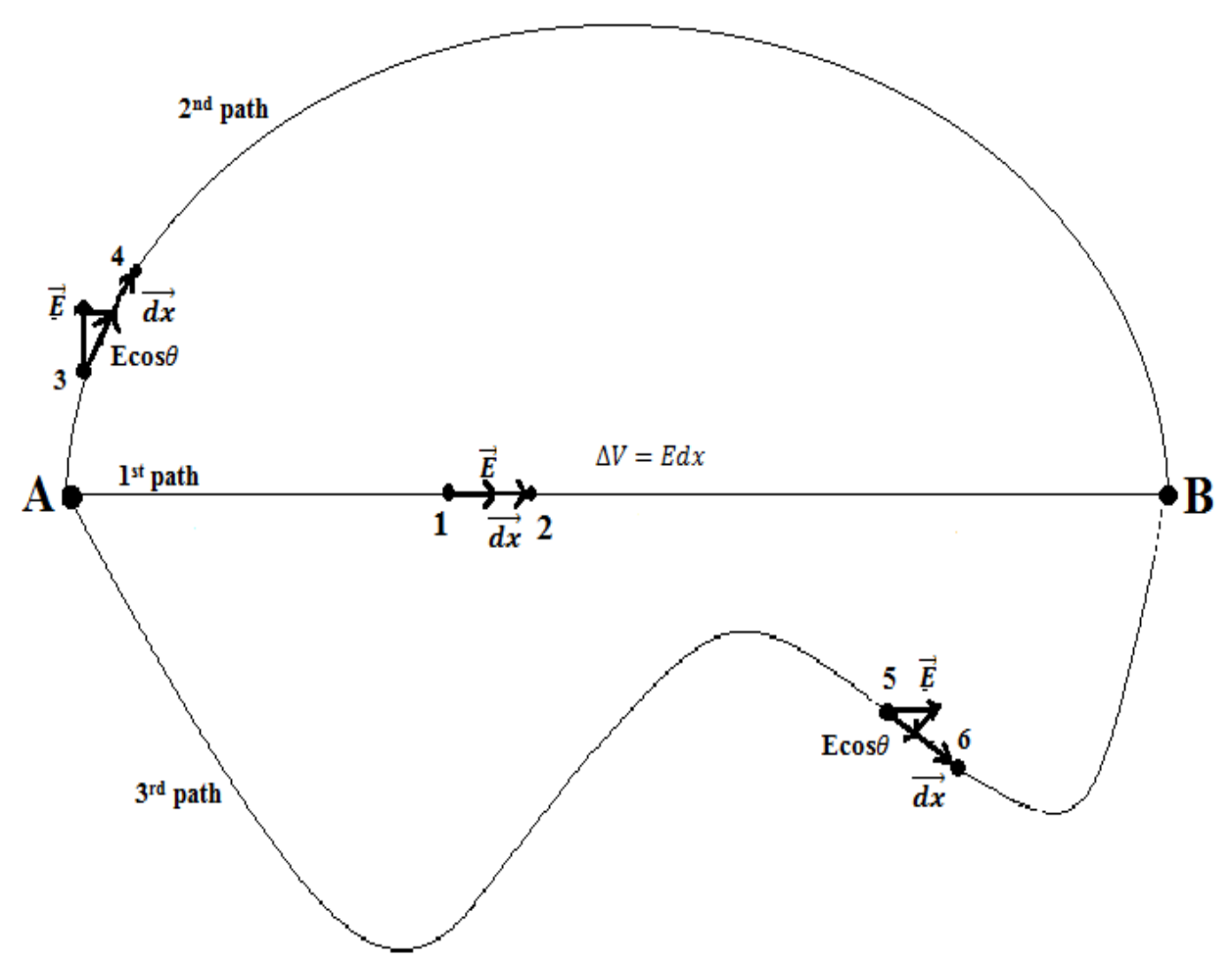

Figure 4. The schematic illustration of the actual modelling for all the paths

In order to answer the third problem case, it is important to underline the results of the first and second situations. The first problem case, for the 1st path, concludes that the voltage difference between any two points is determined by the displacement interval and the electric field component on the path, the Equation 2. The second problem case, for the 2nd path, concludes that the overall potential difference between two points, here $A$ and $B$, is equal to the sum of the potential differences measured across sequentially tiny distances that is, $\Delta x$, the Equation 2. Combining the Equations 1 and 2 leads to the equation of,

$V_{B}-V_{A}=\sum_{n} E_{n}^{x} \Delta x_{n}$

where $\Delta x_{n}$ denotes the $\mathrm{n}$. displacement interval and $E_{n}^{x}=E_{n} \cos \theta_{n}$, denotes the electric field component on the actual path for the $\mathrm{n}$. path interval. It is important to note, at this stage, that all the quantities namely, electric field vector, $\vec{E}$, path displacement vector, $\overrightarrow{\Delta x}$, and the actual angle between them, $\theta$, are all varying continuously on the path. Therefore, the electrical field vector component on the path can simply be written as, $E_{n}^{x}=E_{n} \cos \theta_{n}$. Substituting this expression in the equation (3) gives,

$V_{B}-V_{A}=\sum_{n} E_{n} \cos \theta_{n} \Delta x_{n}$

Using the integral definition, that is; if one takes the limit of the equation (4) while $\Delta x_{n} \rightarrow 0$ then the equation (4) transforms to an integral equation, which is,

$V_{B}-V_{A}=\lim _{\Delta x_{n} \rightarrow 0}\left[\sum_{n} E_{n} \cos \theta_{n} \Delta x_{n}\right]=\int E \cos \theta d x$

Finally using the definition of the scalar product of two vectors leads to the final and smart equation of,

$V_{B}-V_{A}=\int \vec{E} \cdot \overrightarrow{d x}$ 
This final equation describes, in general, the potential difference between the points of $A$ and $B$ within a non-uniform electric field, which is the clear answer for the problem statement of 3 . (Beichner \& Serway, 2000; Sears et al., 2016).

\section{Conclusions}

The present work essentially offers a basic priceless and accessible method to realize and demonstrate the tough definition of electric potential difference in a non-uniform electric field. The procedure outlined here basically employs the mathematical modelling which is a popular approach and used for instructing the topic of electric potential difference. This work is also fresh due to developing a basic 3D teaching material, a simple wet cardboard, and outlines all the necessary information for teaching or more specifically mathematical modelling activities. The approach proposed presently is limited to only teaching activities, therefore any further insights concerning the details of the electrical conductance or the mobility of the charges and the effects of the actual cardboard material or detailed chemical properties of the water is beyond the scope of the work. However, we do believe that the method and the 3D material offer a novel and powerful proposal for any physics educator. Especially the 3D wet cardboard, that is very reachable and easy to use material, makes a great advantage for the students and indeed teachers to create a rich and beneficial teaching circumstances.

\section{References}

Afra, N. C., Osta, I., \& Zoubeir, W. (2009). Students' alternative conceptions about electricity and effect of inquiry-based teaching strategies. International Journal of Science and Mathematics Education, 7(1), 103-132. https://doi.org/10.1007/s10763-007-9106-7

Beichner, R. J., \& Serway, R. A. (2000). Physics for scientists and engineers with modern physics. Saunders College Publishing.

Brewe, E. (2008). Modeling theory applied: Modeling Instruction in introductory physics. American Journal of Physics, 76(12), 1155-1160. https://doi.org/10.1119/1.2983148

Carlton, K. (1999). Teaching electric current and electrical potential. Physics Education, 34(6), 341345. https://doi.org/10.1088/0031-9120/34/6/401

Cohen, R., Eylon, B., \& Ganiel, U. (1983). Potential difference and current in simple electric circuits: A study of students' concepts. American Journal of Physics, 51(5), 407-412. https://doi.org/10.1119/1.13226

Gilbert, J. K. (2004). Models and modelling: Routes to more authentic science education. International Journal of Science and Mathematics Education, 2(2), 115-130. https://doi.org/10.1007/s10763-004-3186-4

Gilbert, J. K., Boulter, C. J., \& Elmer, R. (2000). Positioning models in science education and in design and technology education. In Developing Models in Science Education (pp. 3-17). Springer Netherlands. https://doi.org/10.1007/978-94-010-0876-1_1

Greca, I. M., \& Moreira, M. A. (2002). Mental, physical, and mathematical models in the teaching and learning of physics. Science Education, 86(1), 106-121. https://doi.org/10.1002/sce.10013

Grosslight, L., Unger, C., Jay, E., \& Smith, C. L. (1991). Understanding models and their use in science: Conceptions of middle and high school students and experts. Journal of Research in Science Teaching, 28(9), 799-822. https://doi.org/10.1002/tea.3660280907

Gunstone, R., Mulhall, P., \& McKittrick, B. (2009). Physics teachers' perceptions of the difficulty of teaching electricity. Research in Science Education, 39(4), 515-538. https://doi.org/10.1007/s11165-008-9092-y

Hand, B., Gunel, M., \& Ulu, C. (2009). Sequencing embedded multimodal representations in a writing to learn approach to the teaching of electricity. Journal of Research in Science Teaching, 46(3), 225-247. https://doi.org/10.1002/tea.20282 
Hestenes, D. (1987). Toward a modeling theory of physics instruction. American Journal of Physics, 55(5), 440-454. https://doi.org/10.1119/1.15129

Hestenes, D. (1997). Modeling methodology for physics teachers. AIP Conference Proceedings, 399, 935-958. https://doi.org/10.1063/1.53196

Kelly, G. J., Druker, S., \& Chen, C. (1998). Students' reasoning about electricity: combining performance assessments with argumentation analysis. International Journal of Science Education, 20(7), 849-871. https://doi.org/10.1080/0950069980200707

Liégeois, L., Chasseigne, G., Papin, S., \& Mullet, E. (2003). Improving high school students' understanding of potential difference in simple electric circuits. International Journal of Science Education, 25(9), 1129-1145. https://doi.org/10.1080/0950069022000017324

Maloney, D. P., O'Kuma, T. L., Hieggelke, C. J., \& Van Heuvelen, A. (2001). Surveying students' conceptual knowledge of electricity and magnetism. American Journal of Physics, 69(S1), S12S23. https://doi.org/10.1119/1.1371296

Martinez, F., Herrero, L. C., \& de Pablo, S. (2011). Project-based learning and rubrics in the teaching of power supplies and photovoltaic electricity. IEEE Transactions on Education, 54(1), 87-96. https://doi.org/10.1109/TE.2010.2044506

Moodley, K., \& Gaigher, E. (2019). Teaching electric circuits: teachers' perceptions and learners' misconceptions. Research in Science Education, 49(1), 73-89. https://doi.org/10.1007/s11165017-9615-5

Prosser, M. (1994). A phenomenographic study of students' intuitive and conceptual understanding of certain electrical phenomena. Instructional Science, 22(3), 189-205. https://doi.org/10.1007/BF00892242

Retnawati, H., Arlinwibowo, J., Wulandari, N., \& Pradani, R. (2018). Teachers' difficulties and strategies in physics teaching and learning that applying mathematics. Journal of Baltic Science Education, 17(1), 120-135. http://www.scientiasocialis.lt/jbse/?q=node/643

Rosenthal, A. S., \& Henderson, C. (2006). Teaching about circuits at the introductory level: An emphasis on potential difference. American Journal of Physics, 74(4), 324-328. https://doi.org/10.1119/1.2173271

Sears, F. W., Zemansky, M. W., \& Young, H. D. (2016). College physics. Addison Wesley Publishing Company.

Taber, K. S., Trafford, T. de, \& Quail, T. (2006). Conceptual resources for constructing the concepts of electricity: the role of models, analogies and imagination. Physics Education, 41(2), 155-160. https://doi.org/10.1088/0031-9120/41/2/006

Tarciso Borges, A., \& Gilbert, J. K. (1999). Mental models of electricity. International Journal of Science Education, 21(1), 95-117. https://doi.org/10.1080/095006999290859

Zhang, C., Wang, H., Liu, Y., \& Jiang, J. (2018). Investigation and the improvement strategy of the inquiry physics experiment teaching in senior high school. American Journal of Physics and Applications, 6(5), 104. https://doi.org/10.11648/j.ajpa.20180605.11 